TRANSACTIONS OF THE

AMERICAN MATHEMATICAL SOCIETY

Volume 353, Number 7 , Pages $2615-2633$

S 0002-9947(01)02800-8

Article electronically published on March 14, 2001

\title{
LINEAR FUNCTIONALS OF EIGENVALUES OF RANDOM MATRICES
}

\author{
PERSI DIACONIS AND STEVEN N. EVANS
}

\begin{abstract}
Let $M_{n}$ be a random $n \times n$ unitary matrix with distribution given by Haar measure on the unitary group. Using explicit moment calculations, a general criterion is given for linear combinations of traces of powers of $M_{n}$ to converge to a Gaussian limit as $n \rightarrow \infty$. By Fourier analysis, this result leads to central limit theorems for the measure on the circle that places a unit mass at each of the eigenvalues of $M_{n}$. For example, the integral of this measure against a function with suitably decaying Fourier coefficients converges to a Gaussian limit without any normalisation. Known central limit theorems for the number of eigenvalues in a circular arc and the logarithm of the characteristic polynomial of $M_{n}$ are also derived from the criterion. Similar results are sketched for Haar distributed orthogonal and symplectic matrices.
\end{abstract}

\section{INTRODUCTION}

For $n \in \mathbb{N}$, let $M_{n}$ be a random $n \times n$ unitary matrix with distribution given by Haar measure on the unitary group. The eigenvalues of $M_{n}$ lie on the unit circle $\mathbb{T}$ of the complex plane $\mathbb{C}$. Write $\Xi_{n}$ for the random measure on $\mathbb{T}$ that places a unit mass at each of the eigenvalues of $M_{n}$. That is, if the eigenvalues are $\left\{\nu_{n 1}, \ldots, \nu_{n n}\right\}$, then $\Xi_{n}(f):=\int_{\mathbb{T}} f d \Xi_{n}=\sum_{j} f\left(\nu_{n j}\right)$. The purpose of this paper is to study the asymptotic behaviour of the measures $\Xi_{n}$ as $n \rightarrow \infty$.

Note that if $f: \mathbb{T} \rightarrow \mathbb{C}$ has Fourier expansion $f\left(e^{i \theta}\right)=\sum_{j \in \mathbb{Z}} \hat{f}_{j} e^{i j \theta}$, then

$$
\Xi_{n}(f)=n \hat{f}_{0}+\sum_{j=1}^{\infty} \hat{f}_{j} \operatorname{Tr}\left(M_{n}^{j}\right)+\sum_{j=1}^{\infty} \hat{f}_{-j} \overline{\operatorname{Tr}\left(M_{n}^{j}\right)}
$$

where $\operatorname{Tr}$ denotes the trace and, for the moment, we are being informal about conditions under which such developments are valid. Questions about the asymptotic behaviour of $c_{n}\left(\Xi_{n}\left(f_{n}\right)-\mathbb{E}\left[\Xi_{n}\left(f_{n}\right)\right]\right)$ for a sequence of test functions $\left\{f_{n}\right\}$ and sequence of norming constants $\left\{c_{n}\right\}$ may therefore be placed in the larger framework of questions about the asymptotic behaviour of $\sum_{j=1}^{\infty}\left(a_{n j} \operatorname{Tr}\left(M_{n}^{j}\right)+b_{n j} \overline{\operatorname{Tr}\left(M_{n}^{j}\right)}\right)$ for arrays of complex constants $\left\{a_{n j}: n \in \mathbb{N}, j \in \mathbb{N}\right\}$ and $\left\{b_{n j}: n \in \mathbb{N}, j \in \mathbb{N}\right\}$.

Received by the editors July 6, 2000 and, in revised form, October 7, 2000.

2000 Mathematics Subject Classification. Primary 15A52, 60B15, 60F05.

Key words and phrases. Random matrix, central limit theorem, unitary, orthogonal, symplectic, trace, eigenvalue, characteristic polynomial, counting function, Schur function, character, Besov space, Bessel potential.

Research of first author supported in part by NSF grant DMS-9504379.

Research of second author supported in part by NSF grants DMS-9504379, DMS-9703845 and DMS-0071468. 
In $\$ 3$ we give a general criterion under which such sequences of linear combinations of traces converge in distribution to normal. The main tool is an exact computation of the joint moments of the random variables $\operatorname{Tr}\left(M_{n}^{j}\right)$ due to Diaconis and Shahshahani DS94] that we recall in \$2]

An immediate consequence of this convergence criterion is that if $f: \mathbb{T} \rightarrow \mathbb{R}$ is a test function such that $\sum_{j}\left|\hat{f}_{j}\right|^{2}|j|<\infty$, then $\Xi_{n}(f)-\mathbb{E}\left[\Xi_{n}(f)\right]$ converges in distribution to a centred normal random variable $\Xi(f)$ with $\mathbb{E}\left[\Xi(f)^{2}\right]=\sum_{j}\left|\hat{f}_{j}\right|^{2}|j|$ (see 95) - a result which follows easily from those in DS94, but which was made explicit in [Joh97. We also consider the multivariate generalisation of this result for a collection of such test functions. These results are used to show that the complex Poisson integrals of the measures $\Xi_{n}$ converge to a Gaussian analytic function on the unit disk with interesting value distribution properties.

More generally, we establish in $\$ 4$ that if the sequence $\left\{\sum_{j=-k}^{k}\left|\hat{f}_{j}\right|^{2} j\right\}_{k \in \mathbb{N}}$ is slowly varying, then

$$
\frac{\Xi_{n}(f)-\mathbb{E}\left[\Xi_{n}(f)\right]}{\sqrt{\sum_{j=-n}^{n}\left|\hat{f}_{j}\right|^{2}|j|}}
$$

converges in distribution to standard normal.

In 96 we recover the results of Wie98 Sos00] on the number of eigenvalues in a fixed arc. We show that if $f$ is the indicator function of the set $\left\{e^{i \theta}: \theta \in[\alpha, \beta]\right\}$, then

$$
\frac{\Xi_{n}(f)-n \frac{\beta-\alpha}{2 \pi}}{\frac{1}{\pi} \sqrt{\log n}}
$$

converges in distribution to a complex standard normal random variable. (Recall that a complex random variable is said to be standard complex normal if the real and imaginary parts are independent centred (real) normal random variables with common variance $\frac{1}{2}$.) Furthermore, we recover the multivariate extension of this result for several intervals in a manner that "explains" the intriguing covariance structure found in Wie98 as a consquence of the Fourier expansions of the indicator functions of the intervals. We also consider the analogous asymptotics for the number of eigenvalues in arcs which shrink in length as the dimension $n$ increases.

Our methods also recover the results [HKO00] (continued from those in [KS00]) on the asymptotic normality of the suitably normalised logarithm of the characteristic polynomial of $M_{n}$. As remarked in [HKO00, this result coupled with an application of the argument principle gives another "explanation" of the covariance structure of Wie98.

The main virtue of our results on linear combinations of traces is their considerable generality and the fact that they follow from rather elementary method-ofmoments arguments. They rely on a small amount of representation theory for the unitary group and avoid the use of analytic tools such as Szegö-type theorems for Toeplitz determinants. The present paper deals with linear functionals of $\Xi_{n}$ of the form $\int f(\theta) \Xi_{n}(d \theta)$, but a similar development is possible for quadratic functionals of the form $\iint f(\theta, \phi) \Xi_{n}(d \theta) \Xi_{n}(d \phi)$ and higher order functionals. Also, our methods are directly applicable to the orthogonal and symplectic groups, given that the requisite moment formulae have been obtained in [DS94] (the technical report of 
A. Ram that was cited in DS94 for the relevant representation theory of these groups has now appeared as Ram95 - see also Ram97). We outline the relevant arguments in 98 .

\section{Moments OF TRACES}

Theorem 2.1. $\quad$ a) Consider $a=\left(a_{1}, \ldots, a_{k}\right)$ and $b=\left(b_{1}, \ldots, b_{k}\right)$ with $a_{j}, b_{j} \in$ $\{0,1, \ldots\}$. Let $Z_{1}, Z_{2}, \ldots, Z_{k}$ be independent standard complex normal random variables. Then for $n \geq\left(\sum_{j=1}^{k} j a_{j}\right) \vee\left(\sum_{j=1}^{k} j b_{j}\right)$,

$$
\begin{aligned}
& \mathbb{E}\left[\prod_{j=1}^{k}\left(\operatorname{Tr}\left(M_{n}^{j}\right)\right)^{a_{j}} \overline{\left(\operatorname{Tr}\left(M_{n}^{j}\right)\right)^{b_{j}}}\right] \\
& \quad=\delta_{a b} \prod_{j=1}^{k} j^{a_{j}} a_{j} !=\mathbb{E}\left[\prod_{j=1}^{k}\left(\sqrt{j} Z_{j}\right)^{a_{j}} \overline{\left(\sqrt{j} Z_{j}\right)^{b_{j}}}\right] .
\end{aligned}
$$

b) For any $j, k$,

$$
\mathbb{E}\left[\operatorname{Tr}\left(M_{n}^{j}\right) \overline{\operatorname{Tr}\left(M_{n}^{k}\right)}\right]=\delta_{j k}(j \wedge n) .
$$

Proof. Part (a) is Theorem 2 in DS94 with a slightly different (and incorrect) condition on $a, b$. For the sake of completeness, we redo the proof in [DS94].

Define the simple power sum symmetric function $p_{j}$ to be the symmetric function $p_{j}\left(x_{1}, \ldots, x_{n}\right)=x_{1}^{j}+\cdots+x_{n}^{j}$. Let $\mu$ be the partition $\left(1^{a_{1}}, 2^{a_{2}}, \ldots, k^{a_{k}}\right)$ of the integer $K=1 a_{1}+2 a_{2}+\cdots+k a_{k}$ and set $p_{\mu}=\prod_{j} p_{j}^{a_{j}}$ to be the corresponding compound power sum symmetric function. Associate $\mu$ with the conjugacy class of the symmetric group on $K$ letters that consists of permutations with $a_{j} j$-cycles for $1 \leq j \leq k$. We have the expansion

$$
p_{\mu}=\sum_{\lambda \vdash K} \chi_{\mu}^{\lambda} s_{\lambda}
$$

where the sum is over all partitions of $K$, the coefficient $\chi_{\mu}^{\lambda}$ is the character of the irreducible representation of the symmetric group associated with the partition $\lambda$ evaluated on the conjugacy class associated with the partition $\mu$, and $s_{\lambda}$ is the Schur function corresponding to the partition $\lambda$ (see I.7.8 of [Mac79]).

Given an $n \times n$ unitary matrix $U$, write $s_{\lambda}(U)$ (resp. $p_{\mu}(U)$ ) for the function $s_{\lambda}$ (resp. $p_{\mu}$ ) applied to the eigenvalues of $U$. Writing $\ell(\lambda)$ for the number of parts of the partition $\lambda$ (that is, the length of $\lambda$ ), the functions $U \mapsto s_{\lambda}(U)$ are irreducible characters of the unitary group when $\ell(\lambda) \leq n$ and $s_{\lambda}(U)=0$ otherwise (see $\S 11.1$ of [Lit58). Thus

$$
\mathbb{E}\left[s_{\lambda}\left(M_{n} \overline{s_{\pi}\left(M_{n}\right)}\right]=\delta_{\lambda \pi} \mathbf{1}(\ell(\lambda) \leq n)\right.
$$


Set $\nu=\left(1^{b_{1}}, 2^{b_{2}}, \ldots, k^{b_{k}}\right)$ and $L=1 b_{1}+2 b_{2}+\cdots+k b_{k}$. We have

$$
\begin{aligned}
\mathbb{E} & {\left[\prod_{j=1}^{k}\left(\operatorname{Tr}\left(M_{n}^{j}\right)\right)^{a_{j}} \overline{\left(\operatorname{Tr}\left(M_{n}^{j}\right)\right)^{b_{j}}}\right] } \\
& =\mathbb{E}\left[p_{\mu}\left(M_{n}\right) \overline{p_{\nu}\left(M_{n}\right)}\right] \\
& =\mathbb{E}\left[\left(\sum_{\lambda \vdash K} \chi_{\mu}^{\lambda} s_{\lambda}\left(M_{n}\right)\right) \overline{\left(\sum_{\pi \vdash L} \chi_{\nu}^{\pi} s_{\pi}\left(M_{n}\right)\right)}\right] \\
& =\delta_{K L} \sum_{\lambda \vdash K} \chi_{\mu}^{\lambda} \overline{\chi_{\nu}^{\lambda}} \mathbf{1}(\ell(\lambda) \leq n) .
\end{aligned}
$$

When $K \leq n$, all partitions of $K$ are necessarily of length at most $n$, and so, by the second orthogonality relation for characters of the symmetric group (see Equation $(4.2 ; 5)$ of [Lit58]), the rightmost term of (2.1) becomes

$$
\delta_{K L} \delta_{\mu \nu} \prod_{j=1}^{k} j^{a_{j}} a_{j} !=\delta_{a b} \prod_{j=1}^{k} j^{a_{j}} a_{j} !,
$$

which coincides with the claimed mixed moment of $\sqrt{j} Z_{j}, 1 \leq j \leq k$, (see Lemma 1 of [DS94]).

Turning to part (b), we have from (2.1) that

$$
\mathbb{E}\left[\operatorname{Tr}\left(M_{n}^{j}\right) \overline{\operatorname{Tr}\left(M_{n}^{k}\right)}\right]=\delta_{j k} \sum_{\lambda \vdash j}\left|\chi_{(j)}^{\lambda}\right|^{2} \mathbf{1}(\ell(\lambda) \leq n),
$$

where $(j)$ is the partition of $j$ consisting of a single part of size $j$. Now $\chi_{(j)}^{\lambda}=0$ unless $\lambda$ is a hook partition (that is, a partition with at most one part of size greater than 1), in which case

$$
\chi_{(j)}^{\lambda}=(-1)^{\ell(\lambda)-1}
$$

(see, for example, Exercise 4.16 in [FH91]). Since there are $j \wedge n$ hook partitions of $j$ of length at most $n$, part (b) follows.

Remark 2.2. It is shown in [Rai97] that the eigenvalues of $M_{n}^{j}$ for $j \geq n$ are distributed as $n$ independent uniform random variables on $\mathbb{T}$.

\section{Linear combinations of traces}

Theorem 3.1. Consider an array of complex constants $\left\{a_{n j}: n \in \mathbb{N}, j \in \mathbb{N}\right\}$. Suppose there exists $\sigma^{2}$ such that

$$
\lim _{n \rightarrow \infty} \sum_{j=1}^{\infty}\left|a_{n j}\right|^{2}(j \wedge n)=\sigma^{2} .
$$

Suppose also that there exists a sequence of positive integers $\left\{m_{n}: n \in \mathbb{N}\right\}$ such that

$$
\lim _{n \rightarrow \infty} m_{n} / n=0
$$


and

$$
\lim _{n \rightarrow \infty} \sum_{j=m_{n}+1}^{\infty}\left|a_{n j}\right|^{2}(j \wedge n)=0 .
$$

Then $\sum_{j=1}^{\infty} a_{n j} \operatorname{Tr}\left(M_{n}^{j}\right)$ converges in distribution as $n \rightarrow \infty$ to $\sigma Z$, where $Z$ is a complex standard normal random variable.

Proof. Recall from Theorem 2.1 that $\mathbb{E}\left[\operatorname{Tr}\left(M_{n}^{j}\right)\right]=0$ and $\mathbb{E}\left[\operatorname{Tr}\left(M_{n}^{j}\right) \overline{\operatorname{Tr}\left(M_{n}^{k}\right)}\right]=$ $\delta_{j k}(j \wedge n)$. Consequently, the series $\sum_{j=1}^{\infty} a_{n j} \operatorname{Tr}\left(M_{n}^{j}\right)$ converges in $L^{2}$ for each $n$ and $\lim _{n \rightarrow \infty} \mathbb{E}\left[\left|\sum_{j=m_{n}+1}^{\infty} a_{n j} \operatorname{Tr}\left(M_{n}^{j}\right)\right|^{2}\right]=0$.

It therefore suffices to show that $\sigma^{-1} \sum_{j=1}^{m_{n}} a_{n j} \operatorname{Tr}\left(M_{n}^{j}\right)$ converges in distribution as $n \rightarrow \infty$ to a complex standard normal random variable. Let $Z_{0}, Z_{1}, Z_{2}, \ldots$ be a sequence of independent complex standard normals. From Theorem 2.1 we know that

$$
\begin{aligned}
\mathbb{E}\left[\left\{\sum_{j=1}^{m_{n}} a_{n j} \operatorname{Tr}\left(M_{n}^{j}\right)\right\}^{\alpha} \overline{\left\{\sum_{j=1}^{m_{n}} a_{n j} \operatorname{Tr}\left(M_{n}^{j}\right)\right\}^{\beta}}\right] \\
=\mathbb{E}\left[\left\{\sum_{j=1}^{m_{n}} a_{n j} \sqrt{j} Z_{j}\right\}^{\alpha} \overline{\left\{\sum_{j=1}^{m_{n}} a_{n j} \sqrt{j} Z_{j}\right\}^{\beta}}\right] \\
=\mathbb{E}\left[\left\{\left(\sum_{j=1}^{m_{n}}\left|a_{n j}\right|^{2} j\right)^{1 / 2} Z_{0}^{\alpha} \overline{\left\{\left(\sum_{j=1}^{m_{n}}\left|a_{n j}\right|^{2} j\right)^{1 / 2} Z_{0}\right\}^{\beta}}\right],\right.
\end{aligned}
$$

provided that $\alpha m_{n} \leq n$ and $\beta m_{n} \leq n$. The result now follows by convergence of moments for complex normal distributions and the assumption that $m_{n} / n \rightarrow 0$.

A straightforward adaptation of the methods in the proof of Theorem 3.1 establishes the following result. The details are left to the reader.

Theorem 3.2. Consider arrays of complex constants $\left\{a_{n j}: n \in \mathbb{N}, j \in \mathbb{N}\right\}$ and $\left\{b_{n j}: n \in \mathbb{N}, j \in \mathbb{N}\right\}$. Suppose there exist $\sigma^{2}, \tau^{2}$, and $\gamma$ such that

$$
\begin{aligned}
& \lim _{n \rightarrow \infty} \sum_{j=1}^{\infty}\left|a_{n j}\right|^{2}(j \wedge n)=\sigma^{2}, \\
& \lim _{n \rightarrow \infty} \sum_{j=1}^{\infty}\left|b_{n j}\right|^{2}(j \wedge n)=\tau^{2},
\end{aligned}
$$

and

$$
\lim _{n \rightarrow \infty} \sum_{j=1}^{\infty} a_{n j} b_{n j}(j \wedge n)=\gamma .
$$

Suppose also that there exists a sequence of positive integers $\left\{m_{n}: n \in \mathbb{N}\right\}$ such that

$$
\lim _{n \rightarrow \infty} m_{n} / n=0
$$


and

$$
\lim _{n \rightarrow \infty} \sum_{j=m_{n}+1}^{\infty}\left(\left|a_{n j}\right|^{2}+\left|b_{n j}\right|^{2}\right)(j \wedge n)=0 .
$$

Then $\sum_{j=1}^{\infty}\left(a_{n j} \operatorname{Tr}\left(M_{n}^{j}\right)+b_{n j} \overline{\operatorname{Tr}\left(M_{n}^{j}\right)}\right)$ converges in distribution as $n \rightarrow \infty$ to $X+i Y$, where $(X, Y)$ is a pair of centred jointly normal real random variables with

$$
\begin{aligned}
& \mathbb{E}\left[X^{2}\right]=\frac{1}{2}\left(\sigma^{2}+\tau^{2}+2 \Re \gamma\right), \\
& \mathbb{E}\left[Y^{2}\right]=\frac{1}{2}\left(\sigma^{2}+\tau^{2}-2 \Re \gamma\right),
\end{aligned}
$$

and

$$
\mathbb{E}[X Y]=\Im \gamma
$$

\section{IntEgRATION AGAINST FUNCTIONS SATISFYING A FOURIER GROWTH CONDITION}

It is immediate from Theorem 2.1 that for each $k \in \mathbb{N}$ the random vector $\left(\operatorname{Tr}\left(M_{n}\right), \operatorname{Tr}\left(M_{n}^{2}\right), \ldots, \operatorname{Tr}\left(M_{n}^{k}\right)\right)$ converges in distribution to $\left(Z_{1}, \sqrt{2} Z_{2}, \ldots, \sqrt{k} Z_{k}\right)$ as $n \rightarrow \infty$, where $Z_{1}, Z_{2}, \ldots, Z_{k}$ are independent standard complex normal random variables. Therefore, if $f(\theta)=\sum_{j=-k}^{k} \hat{f}_{j} e^{i j \theta}$ is a real-valued trigonometric polynomial, so that $\hat{f}_{-j}=\overline{\hat{f}}_{j}$, then

$$
\begin{aligned}
\Xi_{n}(f)-\mathbb{E}\left[\Xi_{n}(f)\right] & =\Xi_{n}(f)-n \hat{f}_{0} \\
& =\sum_{j=1}^{k}\left[\hat{f}_{j} \operatorname{Tr} M_{n}^{j}+\overline{\hat{f}}_{j} \overline{\operatorname{Tr}\left(M_{n}^{j}\right)}\right] \\
& =2 \sum_{j=1}^{k}\left[\left(\Re \hat{f}_{j}\right)\left(\Re \operatorname{Tr}\left(M_{n}^{j}\right)\right)-\left(\Im \hat{f}_{j}\right)\left(\Im \operatorname{Tr}\left(M_{n}^{j}\right)\right)\right]
\end{aligned}
$$

converges in distribution as $n \rightarrow \infty$ to a centred normal with variance

$$
4 \sum_{j=1}^{k}\left[\frac{j}{2}\left(\Re \hat{f}_{j}\right)^{2}+\frac{j}{2}\left(\Im \hat{f}_{j}\right)^{2}\right]=\sum_{j=-k}^{k}\left|\hat{f}_{j}\right|^{2}|j| .
$$

This observation suggests that $\Xi_{n}(f)-\mathbb{E}\left[\Xi_{n}(f)\right]$ should converge to normal when $f$ is no longer just a trigonometric polynomial but more generally when $f$ is a realvalued function of the form $f(\theta)=\sum_{j \in \mathbb{Z}} \hat{f}_{j} e^{i j \theta}$ with $\sum_{j \in \mathbb{Z}}\left|\hat{f}_{j}\right|^{2}|j|<\infty$. We find in the $\$ 5$ that this is indeed the case (see Theorem 5.1).

First, however, we establish a more general result that shows that even when

$$
\sum_{j \in \mathbb{Z}}\left|\hat{f}_{j}\right|^{2}|j|=\infty
$$

it can still sometimes be the case that

$$
\left(\Xi_{n}(f)-\mathbb{E}\left[\Xi_{n}(f)\right]\right) / \operatorname{Var}\left[\Xi_{n}(f)\right]
$$

is asymptotically normal. 
Given $f \in L^{2}(\mathbb{T})$ (where we define $L^{2}(\mathbb{T})$ to be the space of real-valued squareintegrable functions), write

$$
\hat{f}_{j}:=\frac{1}{2 \pi} \int e^{-i j \theta} f(\theta) d \theta, \quad j \in \mathbb{Z}
$$

for the Fourier coefficients of $f$.

Recall that a positive sequence $\left\{c_{k}\right\}_{k \in \mathbb{N}}$ is said to be slowly varying if

$$
\lim _{k \rightarrow \infty} \frac{c_{\lfloor\lambda k\rfloor}}{c_{k}}=1, \quad \lambda>0,
$$

(see [Fel71, BGT87]).

Theorem 4.1. Suppose that $f \in L^{2}(\mathbb{T})$ is such that $\left\{\sum_{j=-k}^{k}\left|\hat{f}_{j}\right|^{2} j\right\}_{k \in \mathbb{N}}$ is slowly varying. Then

$$
\frac{\Xi_{n}(f)-\mathbb{E}\left[\Xi_{n}(f)\right]}{\sqrt{\sum_{j=-n}^{n}\left|\hat{f}_{j}\right|^{2}|j|}}
$$

converges in distribution to a standard normal random variable as $n \rightarrow \infty$.

Proof. Note first of all for any Borel subset $A \subseteq \mathbb{T}$ that the probability $M_{n}$ has at least one eigenvalue in $A$ (that is, $\mathbb{P}\left\{\Xi_{n}(A) \neq 0\right\}$ ) is, by symmetry, at most $n \frac{1}{2 \pi} \int_{A} d \theta$. Therefore, $\Xi_{n}(f)$ only depends on the $L^{2}(\mathbb{T})$ equivalence class of $f$. Similarly, we have that

$$
\Xi_{n}(f)=\lim _{k \rightarrow \infty} \int \sum_{j=-k}^{k} \hat{f}_{j} e^{i j \theta} \Xi_{n}(d \theta)
$$

in probability, because

$$
f(\theta)=\lim _{k \rightarrow \infty} \sum_{j=-k}^{k} \hat{f}_{j} e^{i j \theta}
$$

in Lebesgue measure. Thus

$$
\Xi_{n}(f)=\sum_{j=1}^{\infty} \hat{f}_{j} \operatorname{Tr} M_{n}^{j}+\sum_{j=1}^{\infty} \overline{\hat{f}_{j}} \overline{\operatorname{Tr} M_{n}^{j}} .
$$

Set $c_{n}=\sum_{j=-n}^{n}\left|\hat{f}_{j}\right|^{2}|j|=2 \sum_{j=1}^{n}\left|\hat{f}_{j}\right|^{2} j$. We will apply Theorem 3.2 with

$$
a_{n j}=\hat{f}_{j} / \sqrt{c_{n}} \quad \text { and } \quad b_{n j}=\overline{\hat{f}}_{j} / \sqrt{c_{n}} .
$$

Because $\left\{c_{n}\right\}_{n \in \mathbb{N}}$ is slowly varying, it is clear that there is a sequence of integers $\left\{m_{n}\right\}_{n \in \mathbb{N}}$ such that $\lim _{n \rightarrow \infty} m_{n}=\infty, \lim _{n \rightarrow \infty} m_{n} / n=0$, and

$$
\lim _{n \rightarrow \infty} c_{m_{n}} / c_{n}=1 \text {. }
$$

Summing by parts,

$$
2 \sum_{j=n+1}^{\infty}\left|\hat{f}_{j}\right|^{2}=\sum_{j=n}^{\infty}\left(c_{j+1}-c_{j}\right) \frac{1}{j+1}=\sum_{j=n+1}^{\infty} c_{j} \frac{1}{j(j+1)}-c_{n} \frac{1}{n+1} .
$$


Note also that

$$
\lim _{n \rightarrow \infty} \frac{n}{c_{n}} \sum_{j=n+1}^{\infty} c_{j} \frac{1}{j(j+1)}=1
$$

by Theorem 1 in $\S$ VIII.9 of [Fel71.

Combining equations (4.1), (4.2), and (4.3) gives

$$
\lim _{n \rightarrow \infty} \sum_{j=m_{n}+1}^{\infty}\left(\left|a_{n j}\right|^{2}+\left|b_{n j}\right|^{2}\right)(j \wedge n)=0 .
$$

Thus the conditions of Theorem 3.2 hold with

$$
\sigma^{2}=\tau^{2}=\gamma=\frac{1}{2}
$$

and this immediately leads to the result.

\section{INTEGRATION AGAINST FUNCTIONS IN $H_{2}^{\frac{1}{2}}$}

Let $H_{2}^{\frac{1}{2}}$ denote the space of functions $f \in L^{2}(\mathbb{T})$ such that

$$
\|f\|_{\frac{1}{2}}^{2}:=\sum_{j \in \mathbb{Z}}\left|\hat{f}_{j}\right|^{2}|j|<\infty
$$

and define an inner product on $H_{2}^{\frac{1}{2}}$ by

$$
\langle f, g\rangle_{\frac{1}{2}}:=\sum_{j \in \mathbb{Z}} \hat{f}_{j} \overline{\hat{g}}_{j}|j|
$$

Alternatively, $H_{2}^{\frac{1}{2}}$ is the space of functions $f \in L^{2}(\mathbb{T})$ such that

$$
\frac{1}{16 \pi^{2}} \iint \frac{(f(\phi)-f(\theta))^{2}}{\sin ^{2}\left(\frac{\phi-\theta}{2}\right)} d \theta d \phi<\infty
$$

and, moreover,

$$
\langle f, g\rangle_{\frac{1}{2}}=\frac{1}{16 \pi^{2}} \iint \frac{(f(\phi)-f(\theta))(g(\phi)-g(\theta))}{\sin ^{2}\left(\frac{\phi-\theta}{2}\right)} d \theta d \phi
$$

(see Equations (1.2.18) and (1.2.21) of [FOT94]).

The space $H_{2}^{\frac{1}{2}}$ is an example of a Bessel-potential function space and it coincides with the Besov space $B_{2,2}^{\frac{1}{2}}$, the Sobolev-Lebesgue space $F_{2,2}^{\frac{1}{2}}$ and the Lipschitz space $\Lambda_{2,2}^{\frac{1}{2}}$ (see Equations (18) and (19) in $\S 3.5 .4$ and Equation (13) in $§ 3.5 .1$ of [ST87]).

Finally, note that if we take the complex Poisson integral of $f \in L^{2}(\mathbb{T})$, namely

$$
\begin{aligned}
\mathcal{P} f(z) & :=\frac{1}{2 \pi} \int \frac{e^{i \theta}+z}{e^{i \theta}-z} f(\theta) d \theta \\
& =\hat{f}_{0}+2 \sum_{j=1}^{\infty} \hat{f}_{j} z^{j}, \quad|z|<1,
\end{aligned}
$$


then, letting $m$ denote Lebesgue measure on the disk $\{z \in \mathbb{C}:|z|<1\}$,

$$
\begin{aligned}
\int\left|\frac{d \mathcal{P} f(z)}{d z}\right|^{2} m(d z) & =\int_{0}^{1} 2 \pi\left[4 \sum_{j=1}^{\infty}\left|\hat{f}_{j}\right|^{2} j^{2} r^{2(j-1)}\right] r d r \\
& =2 \pi \sum_{j \in \mathbb{Z}}\left|\hat{f}_{j}\right|^{2} j .
\end{aligned}
$$

Thus, $f \in H_{2}^{\frac{1}{2}}$ if and only if

$$
\int\left|\frac{d \mathcal{P} f(z)}{d z}\right|^{2} m(d z)<\infty
$$

and

$$
\langle f, g\rangle_{\frac{1}{2}}=\frac{1}{2 \pi} \int \frac{d \mathcal{P} f(z)}{d z} \frac{\overline{d \mathcal{P} g(z)}}{d z} m(d z), \quad f, g \in H_{2}^{\frac{1}{2}} .
$$

Theorem 5.1. If $f_{1}, \ldots, f_{k} \in H_{2}^{\frac{1}{2}}$ with $\mathbb{E}\left[\Xi_{n}\left(f_{h}\right)\right]=n \int f_{j}(\theta) d \theta=0$ for $1 \leq h \leq$ $k$, then the random vector $\left(\Xi_{n}\left(f_{1}\right), \ldots, \Xi_{n}\left(f_{k}\right)\right)$ converges in distribution to a jointly normal, centred random vector $\left(\Xi\left(f_{1}\right), \ldots, \Xi\left(f_{k}\right)\right)$ with $\mathbb{E}\left[\Xi\left(f_{h}\right) \Xi\left(f_{\ell}\right)\right]=\left\langle f_{h}, f_{\ell}\right\rangle_{\frac{1}{2}}$.

Proof. Employing the Cramér-Wold device (see Theorem 2.9.5 of [Dur96]), it is enough to consider the case $k=1$. In this case, however, the result is immediate from Theorem 4.1

Remark 5.2. A function in $H_{2}^{\frac{1}{2}}$ need not be a.e. equal to a continuous or even bounded function (see $\S 3.5 .4$ of [ST87]). However, note that if $f \in H_{2}^{\frac{1}{2}}$, then, by (5.1) and Markov's inequality, the Lebesgue measure of the set $\{(\theta, \phi): \mid f(\phi)-$ $f(\theta)|>A| \sin ((\phi-\theta) / 2) \mid\}$ converges to 0 as $A \rightarrow \infty$. In particular, a function in $H_{2}^{\frac{1}{2}}$ cannot have jump discontinuities.

Remark 5.3. It is immediate from (5.1) that if $f \in H_{2}^{\frac{1}{2}}$ and $\varphi: \mathbb{R} \rightarrow \mathbb{R}$ is Lipschitz (that is, $|\varphi(x)-\varphi(y)| \leq K|x-y|$ for some constant $K$ ), then $\phi \circ f \in H_{2}^{\frac{1}{2}}$.

Remark 5.4. The space $H_{2}^{\frac{1}{2}}$ equipped with the inner product $\langle\cdot, \cdot\rangle_{\frac{1}{2}}$ is nothing other than the Dirichlet space and Dirichlet form of the symmetric Cauchy process on the circle (see Example 1.4.2 of FOT94]). (The symmetric Cauchy process on the circle is just the usual symmetric Cauchy process on the line wrapped around the circle.)

This observation can be used to deduce various features of the Gaussian process $\left\{\Xi(f): f \in H_{2}^{\frac{1}{2}}, \int f=0\right\}$, several of which will be in a forthcoming paper of the authors. We merely point out for the moment that this fact has consequences for the continuity properties of functions in $H_{2}^{\frac{1}{2}}$. There is a natural notion of capacity associated with $\langle\cdot, \cdot\rangle_{\frac{1}{2}}$, and any function in $H_{2}^{\frac{1}{2}}$ has a quasi-continuous modification with respect to this capacity. That is, if $f \in H_{2}^{\frac{1}{2}}$, then there exists $g$ such that $g=f$ a.e. and for any $\epsilon>0$ there is an open set $G$ of capacity less than $\epsilon$ such that $g$ restricted to $\mathbb{T} \backslash G$ is continuous (see Theorem 2.1.3 of [FOT94]). We note that the capacity associated with $\langle\cdot, \cdot\rangle_{\frac{1}{2}}$ appearing in this definition can be replaced by the classical logarithmic capacity on $\mathbb{T}$. 
Probabilistically, quasi-continuity can be characterised as follows. Write $\left(X_{t}, \mathbb{P}^{x}\right)$ for the symmetric Cauchy process on the circle. A function $g$ is quasi-continuous if there exists a set $N \subset \mathbb{T}$ such that $\mathbb{P}^{x}\left\{\exists t>0: X_{t} \in N\right\}=0$ for all $x \in \mathbb{T}$ and

$$
\mathbb{P}^{x}\left\{\lim _{s \downarrow t} g\left(X_{s}\right)=g\left(X_{t}\right) \text { and } \lim _{s \uparrow t} g\left(X_{s}\right)=g\left(X_{t-}\right), \quad \forall t \geq 0\right\}=1
$$

for all $x \notin N$.

It is certainly not the case that quasi-continuity is a sufficient condition for membership in $H_{2}^{\frac{1}{2}}$. For example, we know from above that the indicator function of an interval $[\alpha, \beta]$ with $0<\alpha<\beta<2 \pi$ is not in $H_{2}^{\frac{1}{2}}$ (this is also obvious by direct computation of Fourier coefficients - see the proof of Theorem 6.1 below). However, because the Cauchy process does not hit points we have

$$
\mathbb{P}^{x}\left\{\exists t>0: X_{t} \in\{\alpha, \beta\} \text { or } X_{t-} \in\{\alpha, \beta\}\right\}=0
$$

for all $x>0$ and hence the indicator function is quasi-continuous.

Remark 5.5. It is immediate that if $f, g \in L^{\infty}(\mathbb{T}) \cap H_{2}^{\frac{1}{2}}$, then the product $f g$ is also in $L^{\infty}(\mathbb{T}) \cap H_{2}^{\frac{1}{2}}$ with $\|f g\|_{\frac{1}{2}} \leq\|f\|_{\infty}\|g\|_{\frac{1}{2}}+\|g\|_{\infty}\|f\|_{\frac{1}{2}}$. This is an instance of a standard fact for Dirichlet forms (see Theorem 1.4.2(ii) of [FOT94]), but it is also immediate from (5.1). The algebra $L^{\infty}(\mathbb{T}) \cap H_{2}^{\frac{1}{2}}$ is known as the Krein algebra and appears in the study of Hankel operators (see $\S 5.2$ of [BS99]).

Example 5.6. Consider the complex Poisson integral of the random measure $\Xi_{n}$, that is, the random analytic function $F_{n}$ on $\{z \in \mathbb{C}:|z|<1\}$ given by

$$
\begin{aligned}
F_{n}(z) & :=\frac{1}{2 \pi} \int \frac{e^{i \theta}+z}{e^{i \theta}-z} \Xi_{n}(d \theta) \\
& =\frac{n}{2 \pi}+\frac{1}{\pi} \sum_{j=1}^{\infty} \overline{\operatorname{Tr}\left(M_{n}^{j}\right)} z^{j} .
\end{aligned}
$$

The harmonic function $\Re F_{n}$ has the measure $\Xi_{n}$ as its "boundary value" in the sense that as $r \uparrow 1$ the measure $\Re F_{n}\left(r e^{i \theta}\right) d \theta$ converges weakly to $\Xi_{n}$ (that is, $\int f(\theta) \Re F_{n}\left(r e^{i \theta}\right) d \theta \rightarrow \int f(\theta) \Xi_{n}(d \theta)$ for all real-valued continuous functions $\left.f\right)$. Note also that

$$
F_{n}(z)=-\frac{1}{\pi} \frac{\chi_{M_{n}}^{\prime}(z)}{\chi_{M_{n}}(z)}-\frac{n}{2 \pi}
$$

where

$$
\chi_{M_{n}}(z):=\operatorname{det}\left(M_{n}-z I\right)
$$

is the characteristic polynomial of $M_{n}$.

It follows from Theorem 5.1 that the finite dimensional distributions of $F_{n}-\frac{n}{2 \pi}$ converge to those of the random analytic function

$$
G(z):=\frac{1}{\pi} \sum_{j=1}^{\infty} \sqrt{j} Z_{j} z^{j}, \quad|z|<1,
$$

where $Z_{1}, Z_{2}, \ldots$ are i.i.d. standard complex normal random variables. Indeed, one can easily check tightness and actually show convergence in distribution in the space of continuous $\mathbb{C}$-valued functions on $\{z \in \mathbb{C}:|z|<1\}$ equipped with the topology of uniform convergence on compacts, but we leave this to the reader. 
Random analytic functions such as $G$ have been much studied (see, for example, Kah85]). For example, let $c(r, b)$ denote the number of zeroes of $G(z)-b$ in $\{z \in \mathbb{C}:|z|<r\}$. For $K \geq 0$ set

$$
C^{*}(K, r):=\sup _{|b| \leq K} \int_{\frac{1}{2}}^{r} \frac{c(s, b)}{s} d s
$$

and

$$
C_{*}(K, r):=\inf _{|b| \leq K} \int_{\frac{1}{2}}^{r} \frac{c(s, b)}{s} d s .
$$

An easy fourth moment calculation and Borel-Cantelli argument shows that

$$
\lim _{r \uparrow 1}(1-r)^{2} \sum_{j=1}^{\infty} j Z_{j}^{2} r^{2 j}=\frac{1}{4} \text { a.s. }
$$

Therefore, by Theorem 1 of Off72 and the remarks after that result we have

$$
\lim _{r \uparrow 1} \frac{C^{*}(K, r)}{\log \left(\frac{1}{1-r}\right)}=\lim _{r \uparrow 1} \frac{C_{*}(K, r)}{\log \left(\frac{1}{1-r}\right)}=1 \text { a.s. }
$$

Consequently, almost surely $G$ takes every value in the complex plane infinitely often. In fact, $G$ restricted to any sector of the unit disk almost surely takes every value infinitely often (see Theorem 2 of [Off72]). In particular, almost surely for each $b \in \mathbb{C}$ every point of the circle $\mathbb{T}$ is a limit point of the level set $\{|z|<1: G(z)=b\}$.

\section{Number of Eigenvalues in AN ARC}

For $0 \leq \alpha<\beta<2 \pi$ write $N_{n}(\alpha, \beta)$ for the number of eigenvalues of $M_{n}$ of the form $e^{i \theta}$ with $\theta \in[\alpha, \beta]$. That is, $N_{n}(\alpha, \beta)=\Xi_{n}(f)$ where $f$ is the indicator function of the arc $\left\{e^{i \theta}: \theta \in[\alpha, \beta]\right\}$. Note that $\mathbb{E}\left[N_{n}(\alpha, \beta)\right]=n(\beta-\alpha) / 2 \pi$. The following result is in Wie98].

Theorem 6.1. As $n \rightarrow \infty$, the finite-dimensional distributions of the processes

$$
\frac{N_{n}(\alpha, \beta)-\mathbb{E}\left[N_{n}(\alpha, \beta)\right]}{\frac{1}{\pi} \sqrt{\log n}}, \quad 0 \leq \alpha<\beta<2 \pi,
$$

converge to those of a centred Gaussian process $\{Z(\alpha, \beta): 0 \leq \alpha<\beta<2 \pi\}$ with the covariance structure

$$
\mathbb{E}\left[Z(\alpha, \beta) Z\left(\alpha^{\prime}, \beta^{\prime}\right)\right]= \begin{cases}1, & \text { if } \alpha=\alpha^{\prime} \text { and } \beta=\beta^{\prime}, \\ \frac{1}{2}, & \text { if } \alpha=\alpha^{\prime} \text { and } \beta \neq \beta^{\prime}, \\ \frac{1}{2}, & \text { if } \alpha \neq \alpha^{\prime} \text { and } \beta=\beta^{\prime}, \\ -\frac{1}{2}, & \text { if } \beta=\alpha^{\prime}, \\ 0, & \text { otherwise. }\end{cases}
$$

Proof. Recall that the indicator function of the interval $[\alpha, \beta]$ has the Fourier expansion

$$
\theta \mapsto \frac{1}{2 \pi}(\beta-\alpha)+\frac{1}{2 \pi i} \sum_{j=1}^{\infty} \frac{e^{-i j \alpha}-e^{-i j \beta}}{j} e^{i j \theta}+\frac{1}{2 \pi i} \sum_{j=1}^{\infty} \frac{e^{i j \beta}-e^{i j \alpha}}{j} e^{-i j \theta}
$$

The result could be established using Theorem 4.1 but it is somewhat more instructive from the point of view of understanding how the covariance of $Z$ arises to proceed by a direct appeal to Theorem 3.2 
It suffices to show for distinct $0 \leq \varphi_{1}, \ldots, \varphi_{k}<2 \pi$ that the real random vector

$$
\frac{1}{2 \pi i \sqrt{\log n}}\left(\sum_{j=1}^{\infty}\left(\frac{e^{-i j \varphi_{\ell}}}{j} \operatorname{Tr}\left(M_{n}^{j}\right)-\frac{e^{i j \varphi_{\ell}}}{j} \overline{\operatorname{Tr}\left(M_{n}^{j}\right)}\right)\right)_{\ell=1}^{k}
$$

converges in distribution to a vector of independent centred Gaussian random variables with common variance $\frac{1}{2 \pi^{2}}$.

Employing the Cramér-Wold device, it suffices in turn to show for real constants $c_{1}, \ldots, c_{k}$ that

$$
\frac{1}{2 \pi i \sqrt{\log n}}\left(\sum_{\ell} c_{\ell}\left(\sum_{j=1}^{\infty} \frac{e^{-i j \varphi_{\ell}}}{j} \operatorname{Tr}\left(M_{n}^{j}\right)-\frac{e^{i j \varphi_{\ell}}}{j} \operatorname{Tr}\left(M_{n}^{j}\right)\right)\right)
$$

converges in distribution to a centred normal random variable with variance

$$
\frac{1}{2 \pi^{2}} \sum_{\ell} c_{\ell}^{2}
$$

We will apply Theorem 3.2 with

$$
\begin{gathered}
a_{n j}=\frac{1}{2 \pi i \sqrt{\log n}} \sum_{\ell} c_{\ell} \frac{e^{-i j \varphi_{\ell}}}{j}, \\
b_{n j}=\bar{a}_{n j}=-\frac{1}{2 \pi i \sqrt{\log n}} \sum_{\ell} c_{\ell} \frac{e^{i j \varphi_{\ell}}}{j},
\end{gathered}
$$

and

$$
m_{n}=\frac{n}{\log n}
$$

It is elementary that

$$
\frac{1}{\log n} \sum_{j=m_{n}+1}^{\infty} \frac{j \wedge n}{j^{2}}=0
$$

and an application of part (a) of Lemma 6.2 below shows that the conditions of Theorem 3.2 hold with

$$
\sigma^{2}=\tau^{2}=\gamma=\frac{1}{4 \pi^{2}} \sum_{\ell} c_{\ell}^{2}
$$

easily leading to the result.

The following elementary result is used in the preceding proof and elsewhere.

Lemma 6.2. $\quad$ a) For $0<\theta<2 \pi$

$$
\lim _{n \rightarrow \infty} \frac{1}{\log n} \sum_{j=1}^{n} \frac{e^{i j \theta}}{j}=0 .
$$

b) For $\theta>0$,

$$
\lim _{T \rightarrow \infty} \frac{1}{\log T} \int_{1}^{T} \frac{e^{i \theta t}}{t} d t=0
$$


Proof. We only prove part (a). The proof of part (b) is similar and is left to the reader. Summing by parts shows that it suffices to establish

$$
\lim _{n \rightarrow \infty} \frac{1}{n} \sum_{j=1}^{n} \exp (i j \theta)=0
$$

but the expression inside the limit on the left-hand side is just

$$
\frac{1}{n} \frac{\exp (i(n+1) \theta)-\exp (i \theta)}{\exp (i \theta)-1}
$$

We now consider the asymptotics of the number of eigenvalues in an arc that shrinks with $n$. Fix a sequence of positive constants $\left\{K_{n}: n \in \mathbb{N}\right\}$. For $0 \leq$ $\alpha<\beta<\infty$ write $\tilde{N}_{n}(\alpha, \beta)$ for the number of eigenvalues of $M_{n}$ of the form $e^{\overline{i \theta}}$ with $\theta \in\left[\alpha / K_{n}, \beta / K_{n}\right]$. Clearly, one cannot expect normal limiting behaviour if $\frac{1}{K_{n}}=O\left(\frac{1}{n}\right)$, but this turns out to be the only restriction.

Theorem 6.3. Suppose that $K_{n} \rightarrow \infty$ and $K_{n} / n \rightarrow 0$ as $n \rightarrow \infty$. As $n \rightarrow \infty$, the finite-dimensional distributions of the processes

$$
\frac{\tilde{N}_{n}(\alpha, \beta)-\mathbb{E}\left[\tilde{N}_{n}(\alpha, \beta)\right]}{\frac{1}{\pi} \sqrt{\log \left(n / K_{n}\right)}}, \quad 0 \leq \alpha<\beta<\infty,
$$

converge to those of a centred Gaussian process $\{\tilde{Z}(\alpha, \beta): 0 \leq \alpha<\beta<\infty\}$ with the covariance structure

$$
\mathbb{E}\left[\tilde{Z}(\alpha, \beta) \tilde{Z}\left(\alpha^{\prime}, \beta^{\prime}\right)\right]= \begin{cases}1, & \text { if } \alpha=\alpha^{\prime} \text { and } \beta=\beta^{\prime}, \\ \frac{1}{2}, & \text { if } \alpha=\alpha^{\prime} \text { and } \beta \neq \beta^{\prime}, \\ \frac{1}{2}, & \text { if } \alpha \neq \alpha^{\prime} \text { and } \beta=\beta^{\prime}, \\ -\frac{1}{2}, & \text { if } \beta=\alpha^{\prime}, \\ 0, & \text { otherwise. }\end{cases}
$$

Proof. By the same Fourier expansion used in the proof of Theorem 6.1 it suffices to show for distinct $0<\varphi_{1}, \ldots, \varphi_{k}<\infty$ that the real random vector

$$
\frac{1}{2 \pi i \sqrt{\log \left(n / K_{n}\right)}}\left(\sum_{j=1}^{\infty}\left(\frac{e^{-i j \varphi_{\ell} / K_{n}}-1}{j} \operatorname{Tr}\left(M_{n}^{j}\right)-\frac{e^{i j \varphi_{\ell} / K_{n}}-1}{j} \overline{\operatorname{Tr}\left(M_{n}^{j}\right)}\right)\right)_{\ell=1}^{k}
$$

converges in distribution to a random vector of the form $\left(W_{0}+W_{1}, W_{0}+W_{1}, \ldots\right.$, $\left.W_{0}+W_{k}\right)$ where $W_{0}, \ldots, W_{k}$ are independent centred Gaussian random variables with common variance $\frac{1}{2 \pi^{2}}$.

Employing the Cramér-Wold device, it suffices in turn to show for real constants $c_{1}, \ldots, c_{k}$ that

$$
\frac{1}{2 \pi i \sqrt{\log \left(n / K_{n}\right)}}\left(\sum_{\ell} c_{\ell}\left(\sum_{j=1}^{\infty} \frac{e^{-i j \varphi_{\ell} / K_{n}}-1}{j} \operatorname{Tr}\left(M_{n}^{j}\right)-\frac{e^{i j \varphi_{\ell} / K_{n}}-1}{j} \overline{\operatorname{Tr}\left(M_{n}^{j}\right)}\right)\right)
$$

converges in distribution to a centred normal random variable with variance

$$
\frac{1}{2 \pi^{2}}\left(\sum_{\ell} c_{\ell}^{2}+\sum_{\ell^{\prime}, \ell^{\prime \prime}} c_{\ell^{\prime}} c_{\ell^{\prime \prime}}\right)
$$


We will apply Theorem 3.2 with

$$
\begin{gathered}
a_{n j}=\frac{1}{2 \pi i \sqrt{\log \left(n / K_{n}\right)}} \sum_{\ell} c_{\ell} \frac{e^{-i j \varphi_{\ell} / K_{n}}-1}{j} \\
b_{n j}=\bar{a}_{n j}=-\frac{1}{2 \pi i \sqrt{\log \left(n / K_{n}\right)}} \sum_{\ell} c_{\ell} \frac{e^{i j \varphi_{\ell} / K_{n}}-1}{j},
\end{gathered}
$$

and

$$
m_{n}=\frac{n}{\log \left(n / K_{n}\right)}
$$

It is elementary that

$$
\frac{1}{\log \left(n / K_{n}\right)} \sum_{j=m_{n}+1}^{\infty} \frac{j \wedge n}{j^{2}}=0
$$

Note that

$$
\begin{aligned}
& \lim _{n \rightarrow \infty} \frac{1}{\log \left(n / K_{n}\right)} \sum_{j=1}^{n}\left(\frac{e^{-i j \varphi_{\ell^{\prime}} / K_{n}}-1}{j}\right)\left(\frac{e^{i j \varphi_{\ell^{\prime \prime}} / K_{n}}-1}{j}\right)(j \wedge n) \\
& \quad=\lim _{n \rightarrow \infty} \frac{1}{\log \left(n / K_{n}\right)} \sum_{j=1}^{n}\left(e^{-i j \varphi_{\ell^{\prime}} / K_{n}}-1\right)\left(e^{i j \varphi_{\ell^{\prime \prime}} / K_{n}}-1\right) \frac{K_{n}}{j} \frac{1}{K_{n}} \\
& \quad=\lim _{n \rightarrow \infty} \frac{1}{\log \left(n / K_{n}\right)} \int_{1}^{n / K_{n}}\left(e^{-i \varphi_{\ell^{\prime}} t}-1\right)\left(e^{i \varphi_{\ell^{\prime \prime}} t}-1\right) \frac{1}{t} d t \\
& \quad= \begin{cases}1, & \text { if } \varphi_{\ell^{\prime}} \neq \varphi_{\ell^{\prime \prime}}, \\
2, & \text { if } \varphi_{\ell^{\prime}}=\varphi_{\ell^{\prime \prime}},\end{cases}
\end{aligned}
$$

by part (b) of Lemma 6.2 ,

Thus the conditions of Theorem 3.2 hold with

$$
\sigma^{2}=\tau^{2}=\gamma=\frac{1}{4 \pi^{2}}\left(\sum_{\ell} c_{\ell}^{2}+\sum_{\ell^{\prime}, \ell^{\prime \prime}} c_{\ell^{\prime}} c_{\ell^{\prime \prime}}\right)
$$

easily leading to the result.

Remark 6.4. The sort of covariance structure for the limiting Gaussian process seen in Theorems 6.1 and 6.3 was also reported for a different ensemble of random unitary matrices in CL95.

\section{AsYmptotics FOR THE CHARACTERISTIC POLYNOMIAL}

We essentially follow the notation and development given in HKOS00] (see also HKO00). For an $n \times n$ unitary matrix $U$, write

$$
\chi_{U}(z):=\operatorname{det}(U-z I), \quad z \in \mathbb{C},
$$

for the characteristic polynomial of $U$ and set

$$
\zeta_{U}(z):=\operatorname{det}\left(I-\frac{1}{z} U\right)=\left(-\frac{1}{z}\right)^{n} \chi_{U}(z), \quad z \neq 0 .
$$

The zeroes of both $\chi_{U}$ and $\zeta_{U}$ are the eigenvalues of $U$ and lie on the unit circle. 
Let log denote the usual branch of the logarithm defined on $\mathbb{C} \backslash\{v \in \mathbb{C}: \Re v \leq 0\}$, so that $\log (1-v)=-\sum_{j=1}^{\infty} v^{j} / j$ for $|v|<1$. Write $w_{1}, \ldots, w_{n}$ for the eigenvalues of $U$ and set

$$
\Lambda_{U}(z):=\sum_{\ell} \log \left(1-\frac{w_{\ell}}{z}\right)
$$

for $z \in \mathbb{C} \backslash\left(\bigcup_{\ell}\left\{w_{\ell} t: 0 \leq t \leq 1\right\}\right)$, so that $\Lambda_{U}(z)=\log \zeta_{U}(z) \bmod 2 \pi i$. Note that

$$
\Lambda_{U}(z)=-\sum_{j=1}^{\infty} \frac{1}{j} \frac{\operatorname{Tr}\left(U^{j}\right)}{z^{j}}
$$

for $|z|>1$.

If $z$ is a fixed point on the unit circle then, almost surely, $z$ is not an eigenvalue of $M_{n}$ and $\Lambda_{M_{n}}(z)$ is defined. Moreover, if $\left\{w_{k}\right\}$ is a fixed sequence with $\left|w_{k}\right|>1$ for all $k$ and $\lim _{k \rightarrow \infty} w_{k}=z$, then $\lim _{k \rightarrow \infty} \Lambda_{U}\left(w_{k}\right)=\Lambda_{U}(z)$. We know from Theorem 2.1 that $\mathbb{E}\left[\operatorname{Tr}\left(M_{n}^{j}\right)\right]=0$ and $\mathbb{E}\left[\operatorname{Tr}\left(M_{n}^{j}\right) \overline{\operatorname{Tr}\left(M_{n}^{k}\right)}\right]=\delta_{j k}(j \wedge n)$. Therefore,

$$
\lim _{k \rightarrow \infty} \Lambda_{M_{n}}\left(w_{k}\right)=-\lim _{k \rightarrow \infty} \sum_{j=1}^{\infty} \frac{1}{j} \frac{\operatorname{Tr}\left(M_{n}^{j}\right)}{w_{k}^{j}}=-\sum_{j=1}^{\infty} \frac{1}{j} \frac{\operatorname{Tr}\left(M_{n}^{j}\right)}{z^{j}}
$$

in $L^{2}$. Consequently,

$$
\Lambda_{M_{n}}(z)=-\sum_{j=1}^{\infty} \frac{1}{j} \frac{\operatorname{Tr}\left(M_{n}^{j}\right)}{z^{j}} .
$$

The following appears in [HKO00] (see also [KS00]) and we refer the reader there for a discussion of the analogy between this result and Selberg's central limit theorem for the Riemann zeta function.

Theorem 7.1. For distinct points $z_{1}, \ldots, z_{k}$ on the unit circle, the random vectors

$$
\frac{1}{\sqrt{\log n}}\left(\Lambda_{M_{n}}\left(z_{1}\right), \ldots, \Lambda_{M_{n}}\left(z_{k}\right)\right)
$$

converge in distribution as $n \rightarrow \infty$ to a vector of independent complex standard normal random variables.

Proof. It suffices by the Cramér-Wold device to show that if $c_{1}, \ldots, c_{k}$ are complex constants, then $\left.\sum_{\ell} c_{\ell} \Lambda_{M_{n}}\left(z_{\ell}\right)\right) / \sqrt{\log n}$ converges in distribution to $\left(\sum_{\ell}\left|c_{\ell}\right|^{2}\right)^{1 / 2} Z$, where $Z$ is a complex standard normal random variable.

An argument similar to the one in the proof of Theorem 6.1 shows that the conditions of Theorem 3.1 hold with

$$
\begin{gathered}
a_{n j}=\frac{\sum_{\ell} c_{\ell} z_{\ell}^{-j}}{j \sqrt{\log n}}, \\
m_{n}=\frac{n}{\log n},
\end{gathered}
$$

and

$$
\sigma^{2}=\sum_{\ell}\left|c_{\ell}\right|^{2}
$$

as required. 
Remark 7.2. Recalling the notation of \$지, one can use the principle of the argument to show that

$$
N_{n}(\alpha, \beta)-\mathbb{E}\left[N_{n}(\alpha, \beta)\right]=\frac{1}{\pi} \Im\left(\Lambda_{M_{n}}(\beta)-\Lambda_{M_{n}}(\alpha)\right)
$$

(see, for example, [HKOS00]). Consequently, Theorem 6.1 follows from Theorem 7.1.

\section{ORTHOGONAL AND SYMPLECTIC MATRICES}

The methods used above in the context of the unitary group are directly applicable to linear functionals of eigenvalues of Haar distributed random matrices in the orthogonal and symplectic groups. The relevant moment formulae were established in DS94 using the thesis work of Arun Ram that has now been published in Ram95, Ram97. We refer to these papers for background and details. Rather than completely redo the above development in the orthogonal and symplectic settings, we just indicate how our methods extend by sketching the following analogue of Theorem 3.1.

Theorem 8.1. Consider an array of real constants $\left\{a_{n j}: n \in \mathbb{N}, j \in \mathbb{N}\right\}$. Suppose there exists $\mu$ and $\sigma^{2}$ such that

$$
\lim _{n \rightarrow \infty} \sum_{j=1}^{\infty} a_{n, 2 j}=\mu \quad \text { and } \quad \lim _{n \rightarrow \infty} \sum_{j=1}^{\infty} a_{n j}^{2}(j \wedge 2 n)=\sigma^{2} .
$$

Suppose also that there exists a sequence of positive integers $\left\{m_{n}: n \in \mathbb{N}\right\}$ such that

$$
\begin{gathered}
\lim _{n \rightarrow \infty} m_{n} / n=0 \\
\lim _{n \rightarrow \infty} \sum_{j=m_{n}+1}^{\infty}\left|a_{n, 2 j}\right|=0, \quad \text { and } \lim _{n \rightarrow \infty} \sum_{j=m_{n}+1}^{\infty} a_{n j}^{2}(j \wedge 2 n)=0 .
\end{gathered}
$$

If $M_{n}$ is a Haar distributed $n \times n$ orthogonal matrix, then $\sum_{j=1}^{\infty} a_{n j} \operatorname{Tr}\left(M_{n}^{j}\right)$ converges in distribution as $n \rightarrow \infty$ to $\mu+\sigma Z$, where $Z$ is a real standard normal random variable. If $M_{n}$ is a Haar distributed $2 n \times 2 n$ symplectic matrix, then $\sum_{j=1}^{\infty} a_{n j} \operatorname{Tr}\left(M_{n}^{j}\right)$ converges in distribution as $n \rightarrow \infty$ to $-\mu+\sigma Z$

Proof. We consider the orthogonal case, leaving the symplectic case to the reader.

We begin with an analogue of Theorem 2.1. Consider non-negative integers $a_{1}, \ldots, a_{k}$. Let $Z_{1}, Z_{2}, \ldots Z_{k}$ be independent standard normal random variables. Put $\eta_{j}$ to be 1 or 0 according to whether $j$ is even or odd. Then

$$
\mathbb{E}\left[\prod_{j=1}^{k} \operatorname{Tr}\left(M_{n}^{j}\right)^{a_{j}}\right]=\mathbb{E}\left[\prod_{j=1}^{k}\left(\sqrt{j} Z_{j}+\eta_{j}\right)^{a_{j}}\right], \quad n \geq 2 \sum_{j=1}^{k} j a_{j},
$$

and

$$
\mathbb{E}\left[\left(\operatorname{Tr}\left(M_{n}^{j}\right)-\eta_{j}\right)\left(\operatorname{Tr}\left(M_{n}^{k}\right)-\eta_{k}\right)\right]=\delta_{j k}(j \wedge 2 n) .
$$

To establish (8.1), first recall from the proof of Theorem 2.1 that $\prod_{j=1}^{k} \operatorname{Tr}\left(M_{n}^{j}\right)^{a_{j}}$ is the power sum symmetric function $p_{\lambda}$ applied to the eigenvalues of $M_{n}$, where $\lambda$ is the partition $\left(1^{a_{1}}, 2^{a_{2}}, \ldots, k^{a_{k}}\right)$. Set $K=1 a_{1}+2 a_{2}+\cdots+k a_{k}$. 
The power sum symmetric functions may be expressed as linear combinations of the characters $\int_{\nu}$ of the orthogonal group. These characters are indexed by partitions $\nu$ with Young diagrams having at most $n$ boxes in the first two columns (that is, $\nu_{1}^{\prime}+\nu_{2}^{\prime} \leq n$, where $\nu^{\prime}$ is the partition conjugate to $\nu$ ). The change of basis coefficients are the characters of the Brauer algebra $B_{K}(n)$. From pp. 186-187 of Ram95] we have

$$
p_{\lambda}\left(x_{1}, \ldots, x_{n}\right)=\sum_{j=0}^{\lfloor K / 2\rfloor} \sum_{\nu \vdash K-2 j} \mathbf{1}\left\{\nu_{1}^{\prime}+\nu_{2}^{\prime} \leq n\right\} \chi_{K, n}^{\nu}(\omega) \int_{\nu}\left(x_{1}, \ldots, x_{n}\right) ;
$$

here for each partition $\nu$ of $K-2 j, 0 \leq j \leq\lfloor K / 2\rfloor$, the coefficient $\chi_{K, n}^{\nu}(\omega)$ is the character of an irreducible representation of $B_{K}(n)$ evaluated at a permutation $\omega$ of $K$ letters with cycle type $\lambda$.

Integrating over the orthogonal group and using orthogonality of characters shows that

$$
\mathbb{E}\left[p_{\lambda}\left(M_{n}\right)\right]=\chi_{K, n}^{\emptyset}(\omega)
$$

where $\emptyset$ is the trivial partition. Observe that if $K$ is odd, then $\mathbb{E}\left[p_{\lambda}\left(M_{n}\right)\right]=0$; whereas if $K=2 m$ is even and $n \geq 2 K$, then Theorem 2.8 of [Ram97] gives

$$
\chi_{2 m, n}^{\emptyset}(\omega)=\prod_{j=1}^{k} f_{j}\left(a_{j}\right)
$$

where

$$
\begin{aligned}
& \text { for } j \text { odd, } \quad f_{j}(a):= \begin{cases}0, & \text { if } a \text { is odd, } \\
(a-1) ! !, & \text { if } a \text { is even, }\end{cases} \\
& \text { for } j \text { even, } \quad f_{j}(a):=\sum_{s=0}^{\lfloor a / 2\rfloor}\left(\begin{array}{c}
a \\
2 s
\end{array}\right)(2 s-1) ! ! j^{s} .
\end{aligned}
$$

Noting that

$$
f_{j}\left(a_{j}\right)=\mathbb{E}\left[\left(\sqrt{j} Z_{j}+\eta_{j}\right)^{a_{j}}\right]
$$

completes the proof of (8.1).

Turning to (8.2), Theorem 6.8 of [Ram95] provides an alternative expression for the simple power sums $p_{j}$ as linear combinations of the characters $\int_{\nu}$. There are several cases to consider.

For $n=2 r+1$ odd the formulae in Ram95] yield the following. If $j \leq r$, then

$$
p_{j}=\mathbf{1}\{j \text { is even }\}+\sum_{\ell=0}^{(r-1) \wedge(j-1)}(-1)^{\ell} \int_{\left(j-\ell, 1^{\ell}\right)} ;
$$

whereas if $j>r$, then

$$
\begin{aligned}
p_{j}= & \mathbf{1}\{j \text { is even and } r \geq(j+1) / 2\}+\sum_{\ell=0}^{r-1}(-1)^{\ell} \int_{\left(j-\ell, 1^{\ell}\right)} \\
& +\sum_{\ell=r}^{(j-1) \wedge(2 r-1)}(-1)^{\ell} \int_{\left(j-\ell, 1^{2 r-\ell}\right)} .
\end{aligned}
$$


Multiplying $p_{j}$ and $p_{k}$, integrating over the orthogonal group, and using the orthogonality of characters now yields ( $\underline{8.2}$ ). A very similar argument handles the case when $n$ is even.

The proof of the theorem is completed using the method of moments just as in Theorem 3.1.

Remark 8.2. The identity (8.1) is a slightly corrected version of Theorem 4 in DS94]. It is not clear how small $n$ can be to have equality of moments. For example, [Dia87] shows that $\mathbb{E}\left[\operatorname{Tr}\left(M_{n}\right)^{a}\right]=\mathbb{E}\left[Z_{1}^{a}\right]$ for $0 \leq a \leq 2 n+1$. The question rests on the semi-simplicity of $B_{K}(n)$, a matter which has not been fully resolved (cf. [HW89, DWH99]).

\section{ACKNOWLEDGEMENT}

We particularly thank Arun Ram for extensive help. We thank Neil O'Connell for making the preprints [HKOS00] and [HKO00 available to us and for inviting us to a meeting at BRIMS sponsored by Hewlett-Packard and Microsoft where we received useful comments on this work.

\section{REFERENCES}

[BGT87] N.H. Bingham, C.M. Goldie, and J.L. Teugels, Regular Variation, Cambridge University Press, Cambridge, 1987. MR 88i:26004

[BS99] A. Böttcher and B. Silbermann, Introduction to Large Truncated Toeplitz Matrices, Springer-Verlag, New York, 1999. MR 2001b:47043

[CL95] O. Costin and J.L. Lebowitz, Gaussian fluctuation in random matrices, Phys. Rev. Lett. 75 (1995), 69-72.

[Dia87] P. Diaconis, Application of the method of moments in probability and statistics, Moments in mathematics (San Antonio, Tex., 1987), Amer. Math. Soc., Providence, RI, 1987, pp. 125-142. MR 89m:60006

[DS94] P. Diaconis and M. Shahshahani, On the eigenvalues of random matrices, J. Appl. Probab. 31A (1994), 49-62. MR 95m:60011

[Dur96] R. Durrett, Probability: Theory and Examples, 2nd ed., Duxbury, Belmont CA, 1996. MR 98m:60001

[DWH99] W.F. Doran, IV, D.B. Wales, and P.J. Hanlon, On the semisimplicity of the Brauer centralizer algebras, J. Algebra 211 (1999), 647-685. MR 99k:16034

[Fel71] W. Feller, An Introduction to Probability Theory and its Applications, 2nd ed., vol. 2, Wiley, New York, 1971. MR 42:5292

[FH91] W. Fulton and J. Harris, Representation Theory: a first course, Springer-Verlag, New York, 1991. MR 93a:20069

[FOT94] M. Fukushima, Y. Oshima, and M. Takeda, Dirichlet Forms and Symmetric Markov Processes, Walter de Gruyter, Berlin, 1994. MR 96f:60126

[HKO00] C.P. Hughes, J.P. Keating, and N. O'Connell, On the characteristic polynomial of a random unitary matrix, Preprint, 2000.

[HKOS00] B.M. Hambly, P. Keevash, N. O'Connell, and D. Stark, The characteristic polynomial of a random permutation matrix, To appear, 2000.

[HW89] P. Hanlon and D. Wales, On the decomposition of Brauer's centralizer algebras, J. Algebra 121 (1989), 409-445. MR 91a:20041a

[Joh97] K. Johansson, On random matrices from the compact classical groups, Ann. of Math. (2) 145 (1997), 519-545. MR 98e:60016

[Kah85] J.-P. Kahane, Some Random Series of Functions, 2nd ed., Cambridge University Press, Cambridge, 1985. MR 87m:60119

[KS00] J.P. Keating and N.C. Snaith, Random matrix theory and $\zeta\left(\frac{1}{2}+i t\right)$, To appear, 2000.

[Lit58] D.E. Littlewood, The Theory of Group Characters and Matrix Representations of Groups, 2nd ed., Clarendon Press, Oxford, 1958. MR 2,3a (1st ed.)

[Mac79] I.G. Macdonald, Symmetric Functions and Hall Polynomials, Clarendon Press, Oxford, 1979. MR 84g:05003 
[Off72] A.C. Offord, The distribution of the values of a random function in the unit disk, Studia Math. 41 (1972), 71-106. MR 46:6513

[Rai97] E.M. Rains, High powers of random elements of compact Lie groups, Probab. Theory Related Fields 107 (1997), 219-241. MR 98b:15026

[Ram95] A. Ram, Characters of Brauer's centralizer algebras, Pacific J. Math. 169 (1995), 173-200. MR 96k:20020

[Ram97] A. Ram, A "second orthogonality relation" for characters of Brauer algebras, European J. Combin. 18 (1997), 685-706. MR 98m:20015

[Sos00] A. Soshnikov, The central limit theorem for local linear statistics in classical compact groups and related combinatorial identities, Ann. Probab. 28 (2000), 1353-1370. CMP 2001:05

[ST87] H.-J. Schmeisser and H. Triebel, Topics in Fourier Analysis and Function Spaces, Wiley, Chichester, 1987. MR 88k:42015a

[Wie98] K.L. Wieand, Eigenvalue distributions of random matrices in the permutation group and compact Lie groups, Ph.D. thesis, Harvard University, 1998.

Department of Mathematics, Stanford University, Building 380, MC 2125, Stanford, CALIFORNIA 94305

E-mail address: diaconis@math.Stanford.edu

Department of Statistics \#3860, University of California at Berkeley, 367 Evans Hall, Berkeley, California 94720-3860

E-mail address: evans@stat.Berkeley.edu 